\title{
Expression of Neural Markers by Undifferentiated Rat Mesenchymal Stem Cells
}

\author{
Dana Foudah, Juliana Redondo, Cristina Caldara, Fabrizio Carini, \\ Giovanni Tredici, and Mariarosaria Miloso
}

\begin{abstract}
Dipartimento di Neuroscienze e Tecnologie Biomediche, Facoltà di Medicina e Chirurgia, Università degli Studi di Milano-Bicocca, Via Cadore 48, 20052 Monza, Italy
\end{abstract}

Correspondence should be addressed to Mariarosaria Miloso, mariarosaria.miloso@unimib.it

Received 14 March 2012; Revised 13 September 2012; Accepted 13 September 2012

Academic Editor: Michael D. Coleman

Copyright (c) 2012 Dana Foudah et al. This is an open access article distributed under the Creative Commons Attribution License, which permits unrestricted use, distribution, and reproduction in any medium, provided the original work is properly cited.

The spontaneous expression of neural markers by mesenchymal stem cells (MSCs) has been considered to be a demonstration of MSCs' predisposition to differentiate towards neural lineages. In view of their application in cell therapy for neurodegenerative diseases, it is very important to deepen the knowledge about this distinctive biological property of MSCs. In this study, we evaluated the expression of neuronal and glial markers in undifferentiated rat MSCs (rMSCs) at different culture passages (from early to late). rMSCs spontaneously expressed neural markers depending on culture passage, and they were coexpressed or not with the neural progenitor marker nestin. In contrast, the number of rMSCs expressing mesengenic differentiation markers was very low or even completely absent. Moreover, rMSCs at late culture passages were not senescent cells and maintained the MSC immunophenotype. However, their differentiation capabilities were altered. In conclusion, our results support the concept of MSCs as multidifferentiated cells and suggest the existence of immature and mature neurally fated rMSC subpopulations. A possible correlation between specific MSC subpopulations and specific neural lineages could optimize the use of MSCs in cell transplantation therapy for the treatment of neurological diseases.

\section{Introduction}

Cellular therapies using mesenchymal stem cells (MSCs) represent a promising approach in regenerative medicine, tissue-engineering, and autoimmune disease treatment. Clinical studies have confirmed the therapeutic potential of MSCs $[1,2]$ (for other clinical indications presently being tested, please see http://www.clinicaltrials.gov/). However, before the approval of clinical trials in humans, it is mandatory to evaluate in vivo the possible risks of MSC delivery and distribution, and to investigate MSC biology in vitro and the molecular mechanisms regulating their proliferation, differentiation, and senescence. The rat model may represent a suitable model for in vivo and in vitro studies since it has physiological characteristics similar to those of human [3]. Moreover, rat MSCs (rMSCs) and human MSCs express some common surface antigens [4].
The growing interest in rMSCs has led to a number of studies in which their biochemical, genomic, immunophenotypic, and differentiation properties have been examined $[5,6]$. An open question remains regarding the rMSCs' ability to differentiate towards a neuronal lineage. Several differentiation protocols have been proposed [7-10] but, despite the fact that neuronal-like morphology and neuronal proteins expression have been observed, until now neuronal functional properties have not been demonstrated $[9,10]$. The finding that undifferentiated rMSCs spontaneously express neural markers has suggested an intrinsic predisposition of rMSCs to differentiate towards a neuronal lineage. However, the literature data regarding spontaneous neural markers' expression by rMSCs are heterogeneous and discordant [7, 9-14]. In view of the potential therapeutic application of MSCs for neurodegenerative diseases, it has become essential to extend what is known about this 
distinctive property of rMSCs. For this, in the present study, we evaluated the spontaneous expression and coexpression of neural markers in undifferentiated rMSCs at different culture passages (from early to late). In addition, the spontaneous expression of mesengenic differentiation markers was assessed.

\section{Material and Methods}

When not specified, chemicals were purchased from SigmaAldrich, St. Louis, MO, USA.

2.1. rMSC Isolation and Culture. rMSCs were collected, after sacrifice (according to the European Directive 86/609/EEC) from femurs and tibias of 10-week-old female Sprague Dawley (Harlan, Italy) by flushing the shafts with $\alpha$-modified Eagle's medium ( $\alpha$-MEM; Lonza, Verviers, Belgium), serumfree, using a syringe with a no. 20 needle. Cells were disaggregated by gentle pipetting several times. The suspension of cells obtained was centrifuged at $500 \mathrm{rcf}$ for $10 \mathrm{~min}$. Precipitated cells were resuspended in $0.84 \% \mathrm{NH}_{4} \mathrm{Cl}$ for 5 min to dissolve erythrocytes and centrifuged again. The cell pellet was resuspended in a culture medium consisting of $\alpha$-MEM with $20 \%(\mathrm{v} / \mathrm{v})$ embryonic stem-cell screened fetal bovine serum (ES-FBS; Hyclone, Logan, UT), $2 \mathrm{mM}$ L-glutamine, $100 \mathrm{U} / \mathrm{mL}$ penicillin, $100 \mu \mathrm{g} / \mathrm{mL}$ streptomycin, and $250 \mu \mathrm{g} / \mathrm{mL}$ fungizone (Lonza, Verviers, Belgium). Cells were counted by using a Burker chamber and trypan blue staining and were plated in $75 \mathrm{~cm}^{2}$ culture flasks.

rMSC cultures were maintained at $37^{\circ} \mathrm{C}$ in a humidified atmosphere containing $5 \% \mathrm{CO}_{2}$. After $48 \mathrm{~h}$, the nonadherent cells were removed, and the cells attaching to the culture flasks were cultured in $\alpha$-MEM plus $20 \%$ (v/v) ES-FBS, with a change of medium every 3-4 days. When rMSC cultures reached $80-90 \%$ confluence, cells were detached by using $0.05 \%(\mathrm{w} / \mathrm{v})$ trypsin in $0.1 \%(\mathrm{w} / \mathrm{v})$ EDTA (Lonza, Verviers, Belgium) and were either used in experiments or replated $(1 / 3)$ in $75 \mathrm{~cm}^{2}$ culture flasks and expanded. To be passaged, cells were washed twice with Dulbecco's phosphate buffer saline (PBS) to remove the serum added to the medium, detached with trypsin/EDTA solution and replated.

2.2. rMSC Characterization. The rMSCs isolated from rat bone marrow were characterized for their immunophenotype by flow cytometry analysis and for their capacity to differentiate towards mesengenic lineages using specific protocols, as described in a previous work [5]. In particular, in this study, rMSC immunophenotype and differentiation capacity were evaluated at late passages as P40 and P80.

rMSCs were characterized for genomic instability at several passages [5]. We evaluated senescence in rMSC cultures at P40, P80, and P140 (the latest culture passage examined of rMSCs, already in culture for expansion) by using the senescence $\beta$-galactosidase staining kit (Cell Signaling Technology, Danvers, MA, USA) according to the manufacturer's instructions. Cells were seeded at a density of $1 \times 10^{4} / \mathrm{cm}^{2}$ and cultured for $24 \mathrm{~h}$ before senescenceassociated $\beta$-galactosidase staining. At the end of the staining procedure, representative images were taken from several areas of each cell culture using phase-contrast microscopy. For the determination of senescent cell percentage in the culture, an average value was calculated by counting, in eight random fields, the total number of cells and the number of cells with intracellular blue dye.

2.3. Immunofluorescence Experiments. rMSCs ( $\mathrm{P} 0, \mathrm{P} 1, \mathrm{P} 2$, $\mathrm{P} 4, \mathrm{P} 8, \mathrm{P} 16, \mathrm{P} 24, \mathrm{P} 40$, and $\mathrm{P} 80$ ) from 5 rats, respectively, were seeded at approximately $10^{4}$ cells/dish on noncoated glass slides into $35 \mathrm{~mm}$ diameter dishes using a culture medium composed of $\alpha$-MEM plus $10 \%(\mathrm{v} / \mathrm{v})$ ES-FBS. Immunofluorescence was performed at days 10, 14, 21 from plating. In coexpression experiments, immunofluorescence was performed at days 14 and 21. (dorsal root ganglia) DRG primary cultures, in which neurons and glial cells were present, were used as controls for neural markers.

Cells were fixed with $4 \%(\mathrm{w} / \mathrm{v})$ paraformaldehyde for $10 \mathrm{~min}$, washed with PBS, and treated for $10 \mathrm{~min}$ with $0.1 \mathrm{M}$ glycine to quench autofluorescence. Then cells were incubated for 1 hour at room temperature with blocking solution $(5 \%(\mathrm{w} / \mathrm{v})$ BSA, $0.6 \%(\mathrm{v} / \mathrm{v})$ Triton X-100 in PBS) and, subsequently, for $30 \mathrm{~min}$ at $37^{\circ} \mathrm{C}$ with $1 \mathrm{mg} / \mathrm{mL}$ RNAse in blocking-solution. Incubation with the following primary antibodies (diluted in blocking solution) was performed overnight at $4^{\circ} \mathrm{C}$ : anti-Nestin $(1: 50$; Chemicon, Temecula, CA, USA); anti- $\beta$ III tubulin $(1: 100$; Covance, Berkeley, CA, USA); anti-NeuN (1:50; Chemicon, Temecula, CA, USA); anti-Neurofilament $(1: 100 ;$ DakoCytomation, Glostrup, Denmark); anti-GFAP (1:100); anti-S100 (1:100; Chemicon, Temecula, CA, USA); anti-Osteopontin (1:100; Santa Cruz Biotechnology, Inc.); anti-Osteocalcin (1:100; Abcam, Cambridge, UK); anti-PPAR $\gamma 2$ (1:500; Abcam, Cambridge, UK). The following day, cells were rinsed with washing buffer (PBS plus $0.3 \%$ Triton X-100) and incubated at room temperature for 1 hour in the dark with appropriate fluorochrome-conjugated secondary antibodies (Alexa Fluor 488, 555 anti-mouse and anti-rabbit, 1:200; Invitrogen, Oregon, USA). Propidium iodide $(2.5 \mu \mathrm{g} / \mathrm{mL})$, as a nuclear marker, or Alexa Fluor 546-conjugated phalloidin $(1: 200$; Invitrogen, Oregon, USA), as a cytoskeleton filamentous actin marker was used. Negative controls, to verify the specificity of the antibodies, were obtained by omitting primary antibodies and incubating only with secondary antibodies. Cells were then washed with PBS (6 washes of 5 min each) and mounted with polyvinyl alcohol. Microscopy analysis was performed with laser confocal microscopy (Radiance 2100; Biorad Laboratories, Hercules, CA, USA). Noise reduction was achieved by Kalman filtering during acquisition.

2.4. Cell Lysates and Immunoblotting Analysis. rMSCs were washed twice with ice-cold PBS, and total cellular extracts were prepared as previously described [15]. To obtain nuclear protein extracts, the Ronca et al. protocol was performed [16]. The protein concentration was determined by the Bradford assay using a Coomassie protein assay reagent kit (Pierce, Rockford, IL, USA) and aliquots were solubilized in Laemmli buffer $5 \mathrm{x}$, boiled for $5 \mathrm{~min}$, and 


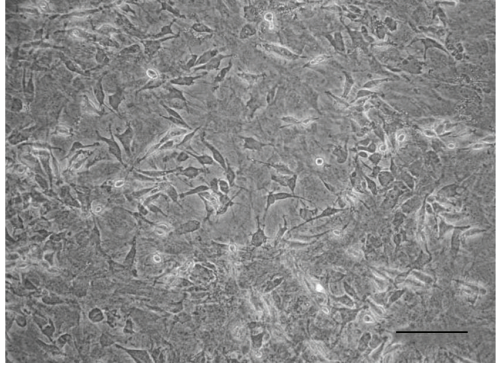

(a)

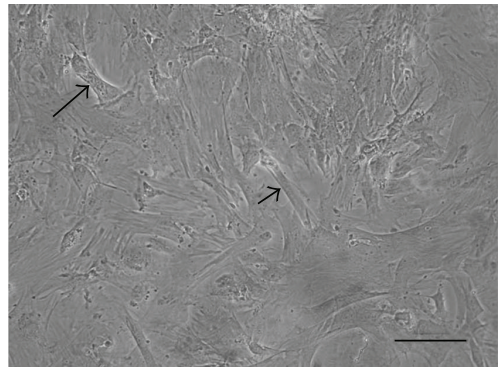

(b)

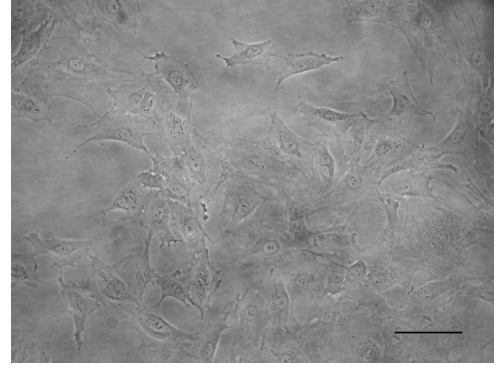

(c)

Figure 1: Cultures of undifferentiated rMSCs at different passages. At P1 rMSC cultures were morphologically heterogeneous (a). At P2 rMSC cultures consisted of a more homogeneous population of cells, most of which had a large flattened morphology (arrow). Relatively elongated cells were also present (arrow head) (b). At P50 rMSCs lost their morphology appearing more rounded (c). Bars:100 $\mu \mathrm{m}$.

run onto $7.5 \%$ or $13 \%$ SDS-PAGE. After electrophoresis, the proteins were transferred to nitrocellulose filters, and immunoblotting analysis was performed. Membrane blocking, washing, and antibody incubation were carried out according to the manufacturer's instructions. Antibodies against nestin $(1: 1000)$, $\beta$ III-tubulin $(1: 3000)$, GFAP $(1: 500)$, and NeuN $(1: 200)$ were used. Anti-actin $(1: 1000$, Santa Cruz, Temecula, CA, USA) immunoblotting analysis was performed as a loading control. After incubation with primary antibodies, membrane was washed and then incubated with appropriate horseradish peroxidas-conjugated secondary antibodies $(1: 2000)$ (anti-mouse, Chemicon, Temecula, CA, USA; anti-rabbit, PerkinElmer, Boston, MA, USA). The immunoreactive proteins were visualized using an ECL chemiluminescence system (Amersham, Arlington Heights, IL, USA). DRG neuron and glial cell total protein extracts were prepared as previously described [15].

2.5. Statistical Analysis. Differences in the number (\%) of cells expressing a specific differentiation marker among passages were analyzed by using one-way analysis of variance (ANOVA). For each marker, an average value of positive cells, after 14 days of culture, was calculated from at least 3 experiments. Data were expressed as mean \pm SD. Comparisons of mean values among the passages were analyzed using a Tukey's multiple comparison test. A five percent probability $(P<0.05)$ was used as the level of significance.

\section{Results}

The present study is an offshoot of a previous work by our group [5]. We used rMSCs derived from the rats examined in the previous study (with the exception of 1 rat), but different parameters have been determined.

3.1. rMSC Isolation and Characterization. The rMSCs, isolated from rat bone marrow and used in our experiments, according to the international criteria proposed for the definition of MSCs [17], were (a) plastic adherent and capable of extensive proliferation when maintained in standard culture conditions; (b) positive for CD29 and CD90 and negative for hematopoietic surface molecules CD34 and CD45; (c) able to differentiate into mesengenic lineages under specific in vitro differentiating conditions [5].

In our culture conditions, rMSCs at P0 and P1 were morphologically heterogeneous (Figure 1(a)). From P2 to P40 rMSC cultures consisted of a more homogeneous population of cells, most of which had a large flattened morphology (Figure 1(b)). Relatively elongated cells were also present (Figure 1(b)). From P40 to subsequent passages, rMSCs lost their flattened morphology appearing more rounded (Figure 1(c)).

rMSCs, at P40 and P80, maintained the immunophenotype observed at P2 [5], being more than $90 \%$ positive for CD29 (Figure 2(a)) and CD90 (Figure 2(b)) and negative for CD34 (Figure 2(c)) and CD45 (Figure 2(d)). However, at P40 and P80, rMSC capacity to differentiate towards mesengenic lineages was altered, with rMSCs being able to differentiate, under appropriate conditions, towards the osteogenic lineage (Figure 2(e)) but not towards the adipogenic one (Figure 2(f)).

In all the culture passages examined rMSCs maintained their capacity to actively divide (data not shown), and rMSCs at P140 are already in culture for expansion. Moreover, as demonstrated by $\beta$-galactosidase staining (Figure 3 ), few senescent cells were present in rMSC cultures at P40, P80, and $\mathrm{P} 140$, where the number of $\beta$-galactosidase-positive cells was respectively, $10 \%, 5 \%$, and $3 \%$.

3.2. Expression of Mesengenic and Neural Markers by Undifferentiated rMSCs. At different passages and at different times (see Section 2) by means of immunofluorescence experiments, we evaluated, in undifferentiated rMSCs cultured in the absence of any differentiative agent, the expression of the following differentiation markers: the neuronal markers $\beta$ III tubulin [18], NeuN [19], and Neurofilament [20]; the glial markers, GFAP [21]; and S100 [22]; the osteogenic markers, osteopontin [23] and osteocalcin [24] and the adipogenic marker, PPAR $\gamma 2$ [25]. Moreover, we analyzed the expression of the neuroprogenitor marker nestin [26]. We estimated the number of cells that were positive for the different markers by considering, for each marker examined, ten fields of 


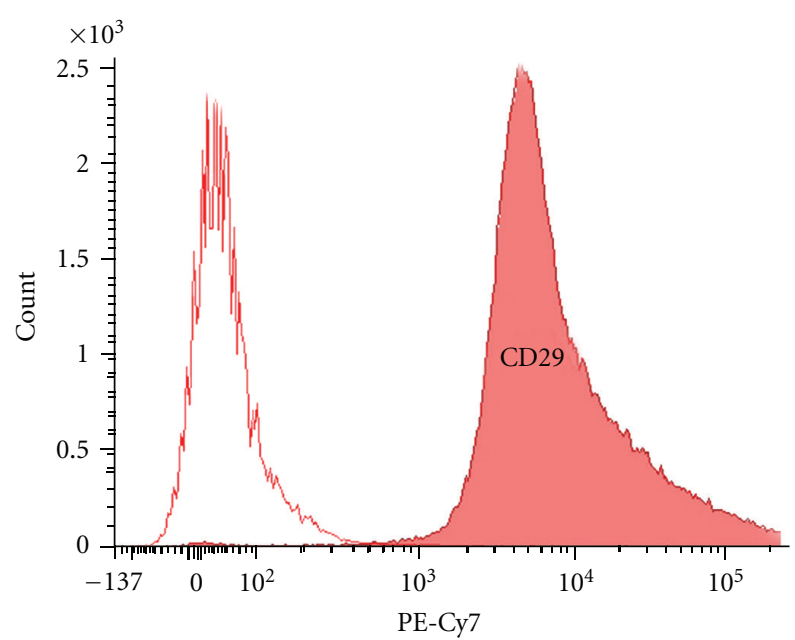

(a)

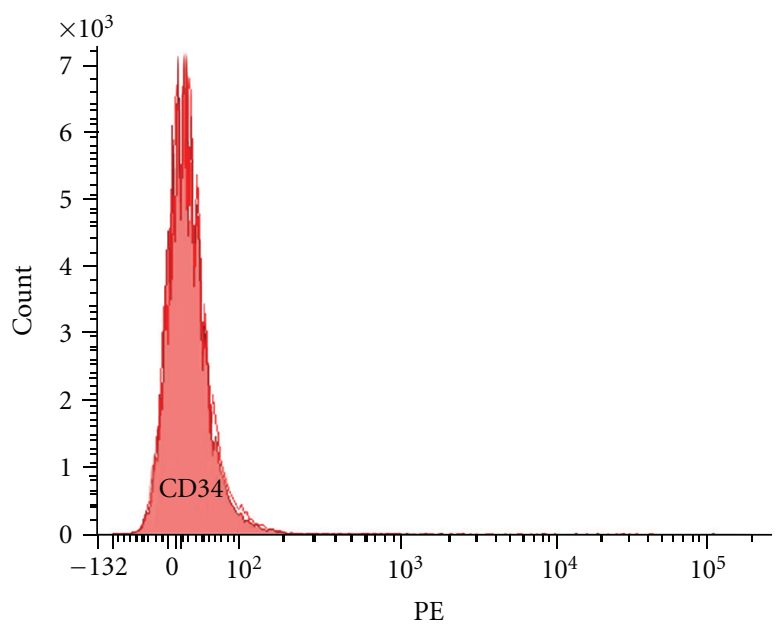

(c)

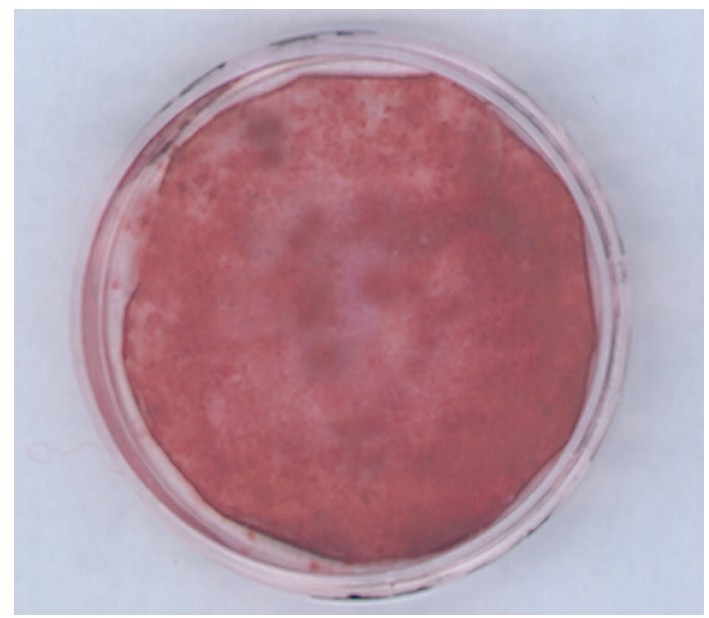

(e)

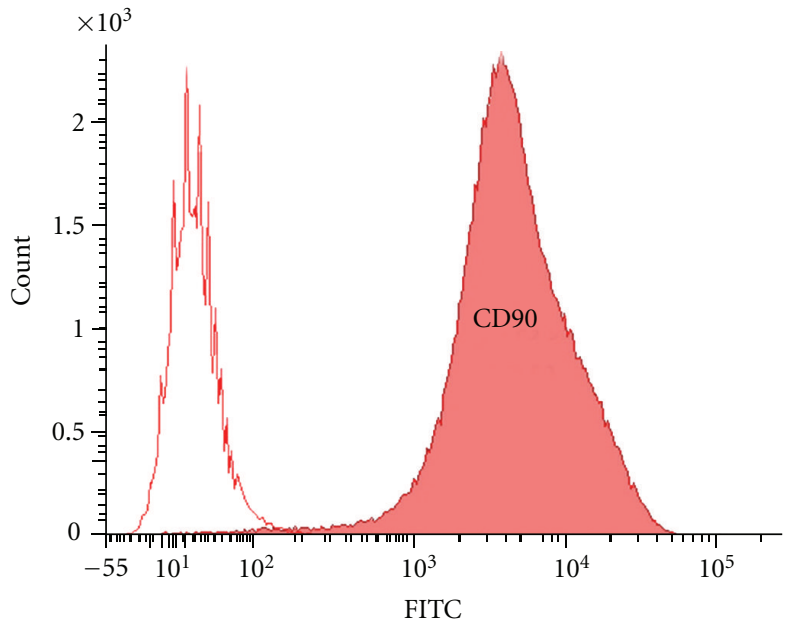

(b)

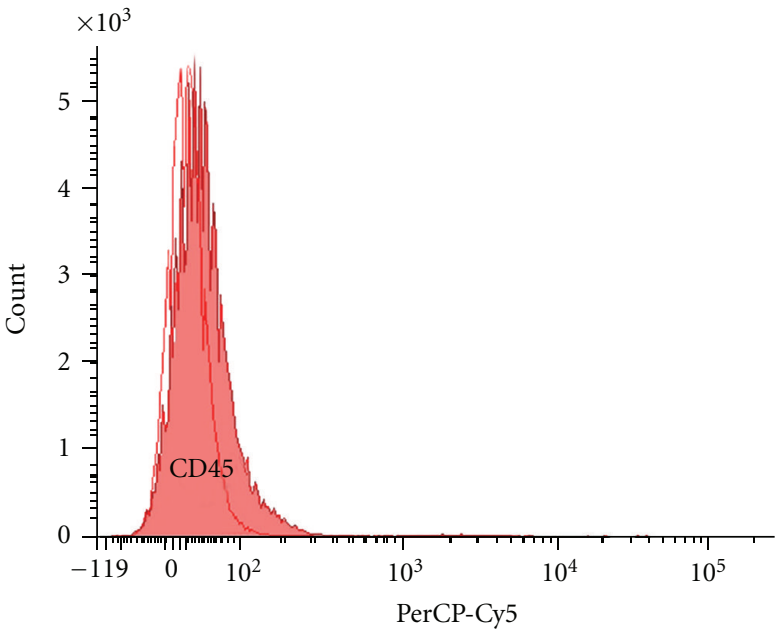

(d)

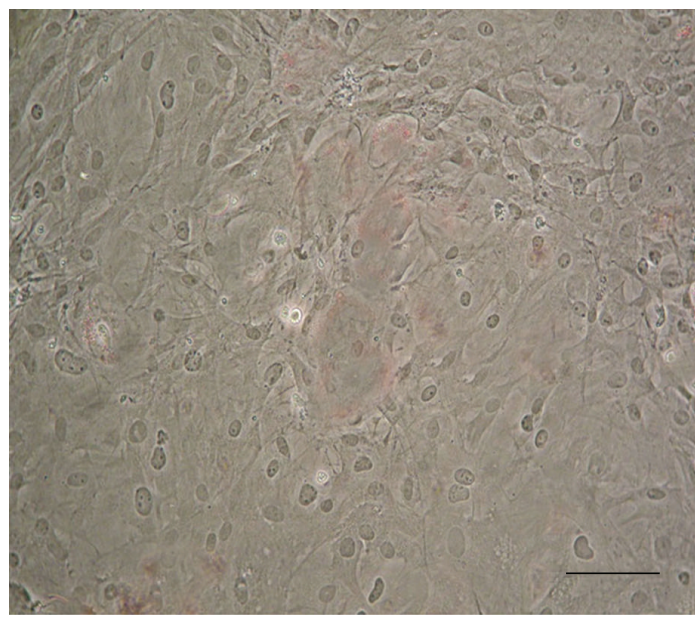

(f)

FIGURE 2: rMSC characterization. (a-d) rMSCs, at P80, were characterized by flow cytometry analysis for the expression of the following markers (pink histograms): CD29 (a), CD90 (b), CD34 (c), and CD45 (d). Isotype-matching IgGs were used to determine nonspecific signals (white histograms). (e and f) rMSC differentiation, at P80, after treatment with specific induction media: osteogenic differentiation was evaluated by alizarin red staining that visualizes calcium deposits (e), adipogenic differentiation was evaluated by oil red $\mathrm{O}$ staining that labels lipid droplets, not observed in adipogenic treated rMSC cultures at P80 (f). Bar: $100 \mu \mathrm{m}$. 


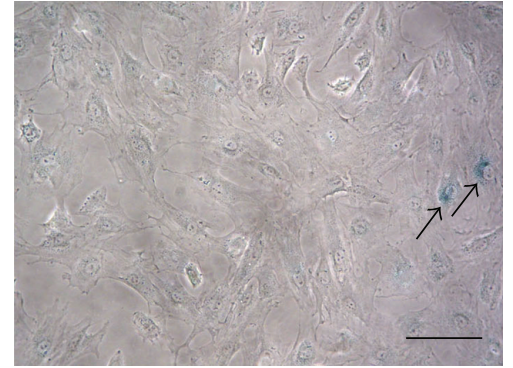

(a)

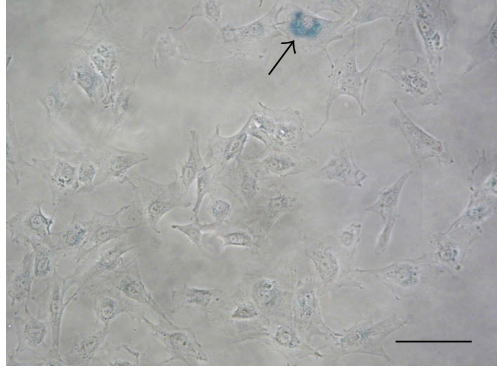

(b)

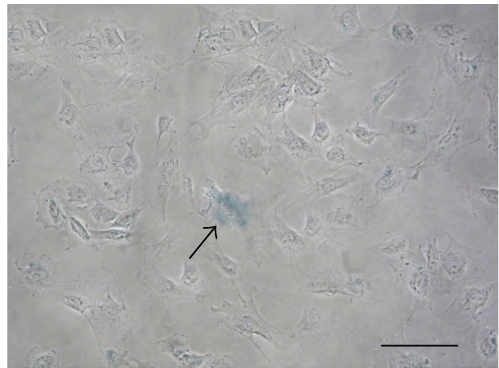

(c)

Figure 3: rMSCs senescence. $\beta$-galactosidase staining (blue, arrow) of rMSCs at P40 (a), P80 (b), and P140 (c). Bars: $100 \mu \mathrm{m}$.

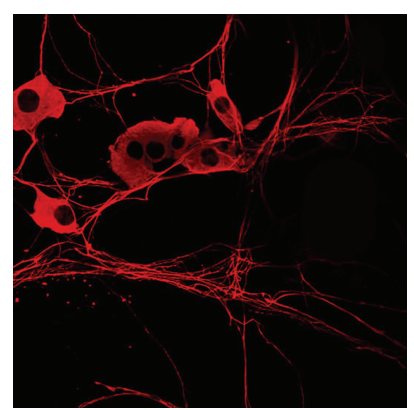

(a)

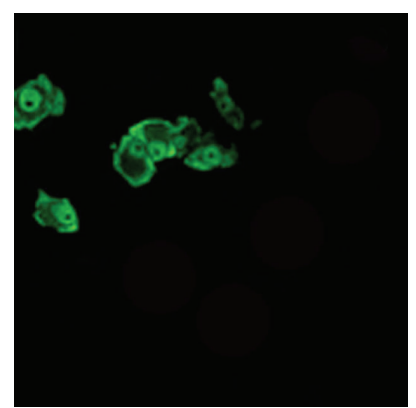

(b)

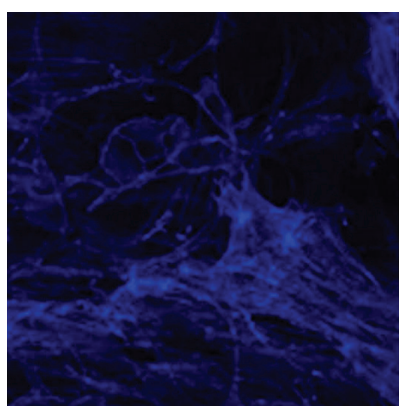

(c)

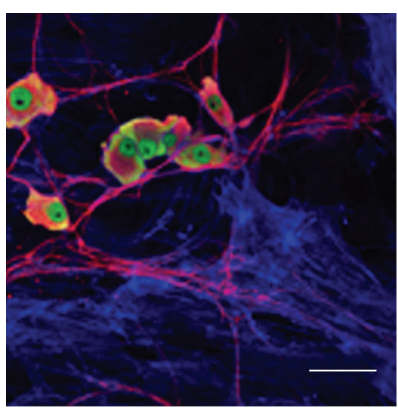

(d)

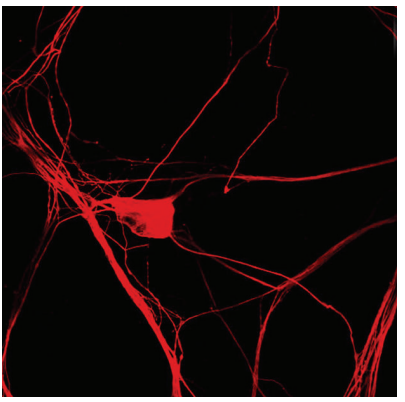

(e)

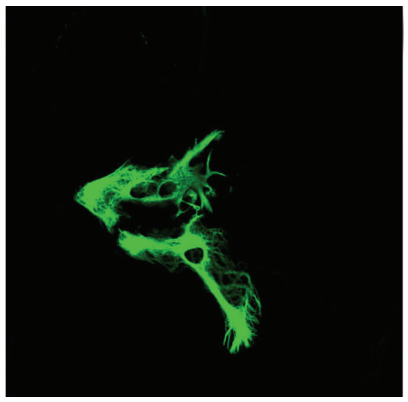

(f)

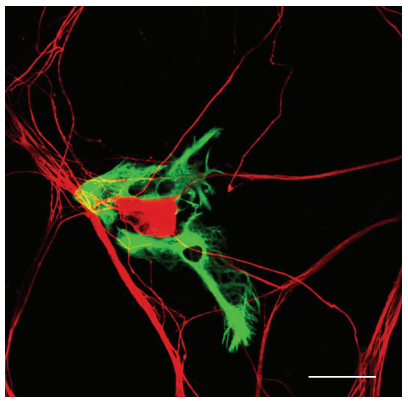

(g)

FIGURE 4: Control cultures represented by DRG primary cultures, in which neurons and glial cells were present. Phalloidin-staining labeled actin filaments in blue (c). Only neurons were positive for $\beta$ III tubulin (a and e, red) and Neun (b, green), and only glial cells were GFAP positive (f, green). Merges ( $\mathrm{d}$ and g). Bars: $50 \mu \mathrm{m}$.

view where positive cells were counted estimating an average value.

The specificity of the antibodies used against neural antigens was confirmed by control cultures represented by DRG primary cultures, in which neurons and glial cells were present (Figure 4). In these control cultures, only neurons were positive for $\beta$ III tubulin (Figures $4(\mathrm{a})$ and $4(\mathrm{e})$ ) and NeuN (Figure 4(b)), and only glial cells were GFAP positive (Figure 4(f)).

The data reported in Table 1 refer to an average value (mean $\pm \mathrm{SD}$ ) of at least 3 experiments for each marker after 14 days of culture. For each culture passage the results remained relatively unchanged for the other time points evaluated. In all the experiments the markers retained their proper cellular localization.

The neuroprogenitor marker nestin was expressed at $\mathrm{P} 0$ and $\mathrm{P} 1$ by about $50 \%$ of rMSCs (Table 1 ). At P2 about $25 \%$ of cells expressed nestin, while at P4, P8, and P16 the percentage of cells that were nestin positive increased considerably reaching about 60\% (Figure 5(b)). At P24 nestin expression decreased (about 30\% positive cells), and at P40 it was drastically reduced and no more than $5 \%$ of cells were nestinpositive. At P80 no nestin-positive cells were present in our rMSC cultures.

At $\mathrm{P} 0$ about $30 \%$ of rMSCs were positive for the early neuronal marker $\beta \mathrm{III}$ tubulin, while at P1, P2, and P4 about 


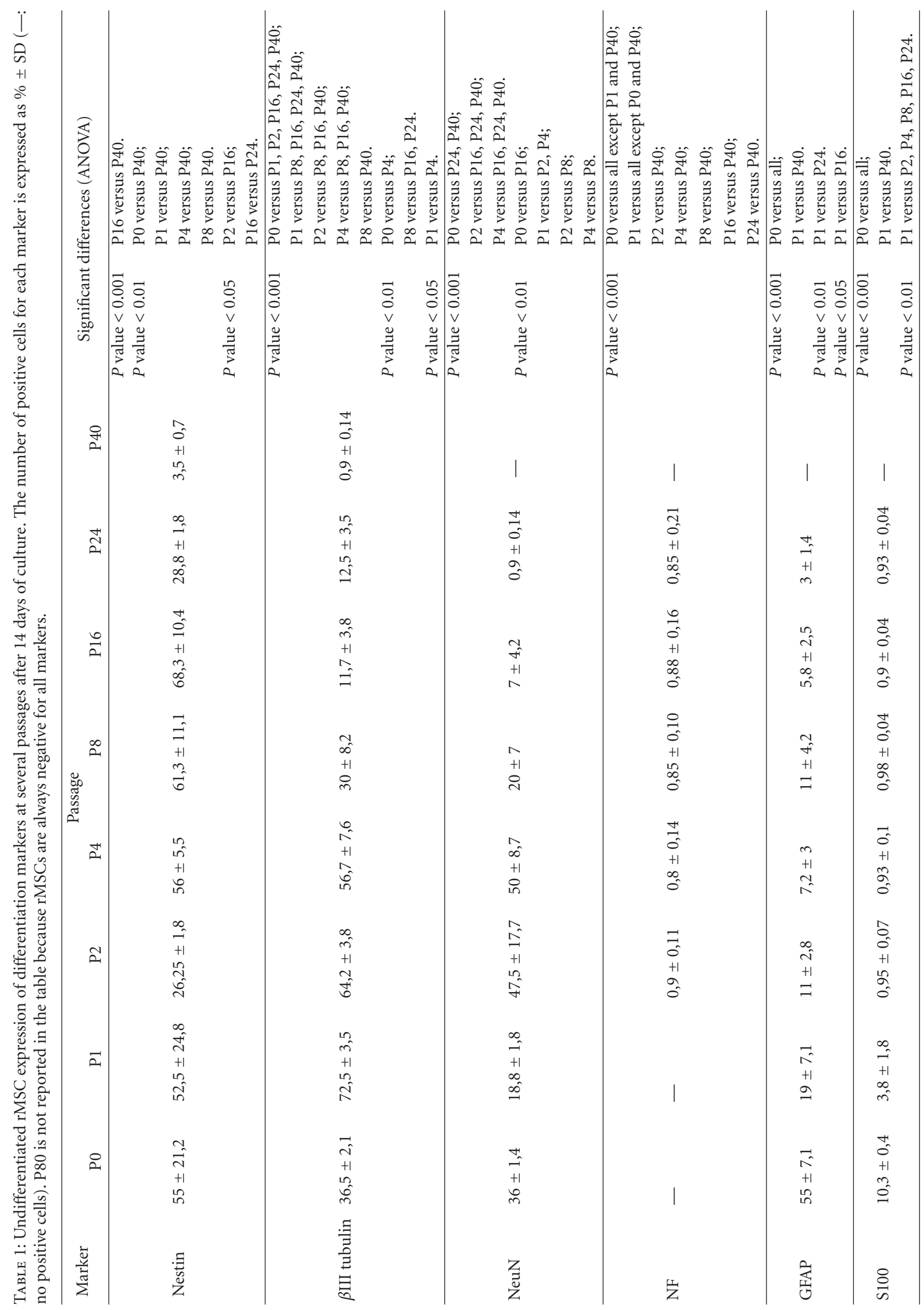




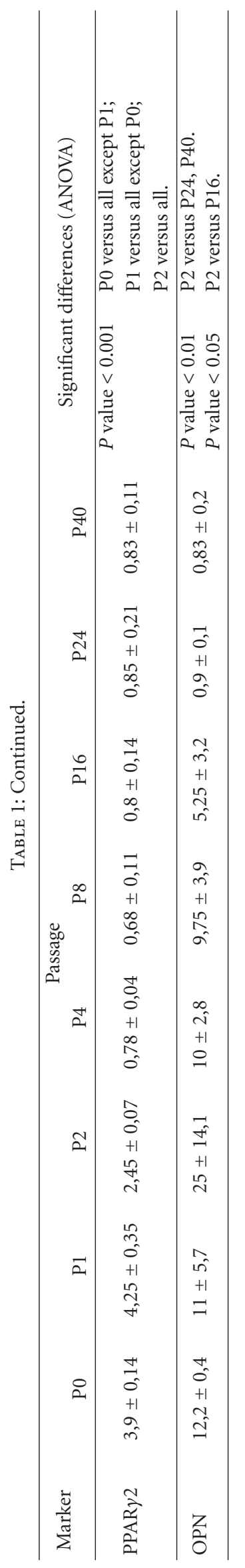




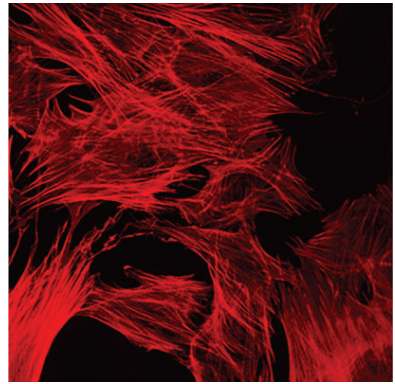

(a)

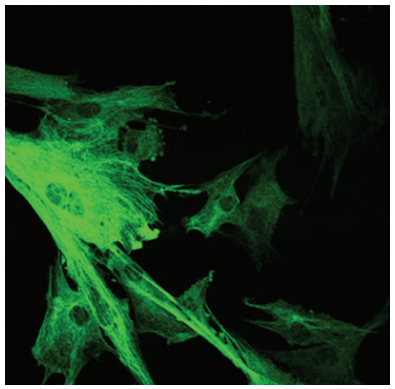

(e)

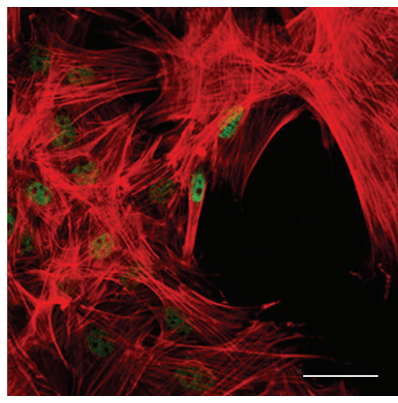

(i)

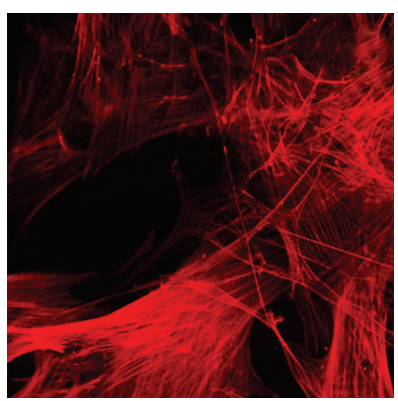

(m)

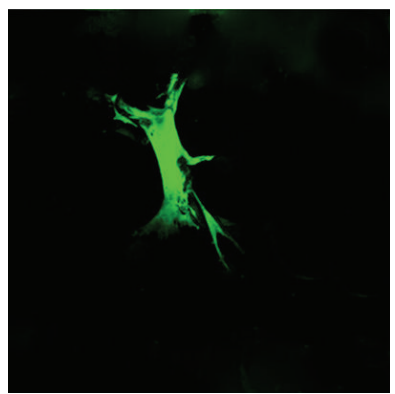

(q)

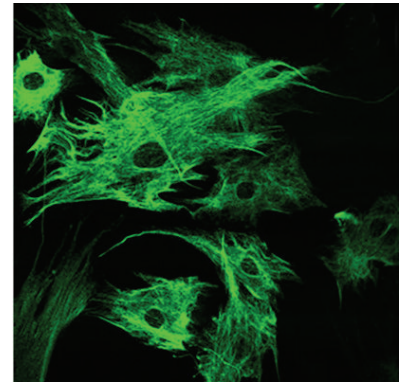

(b)

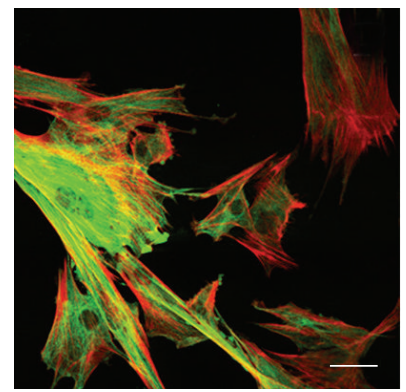

(f)

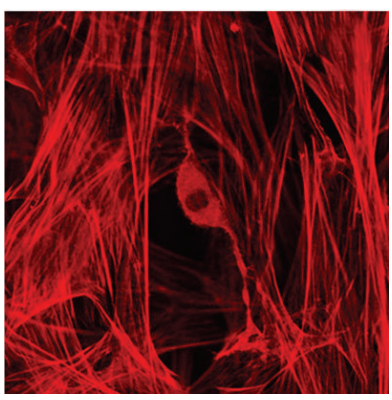

(j)

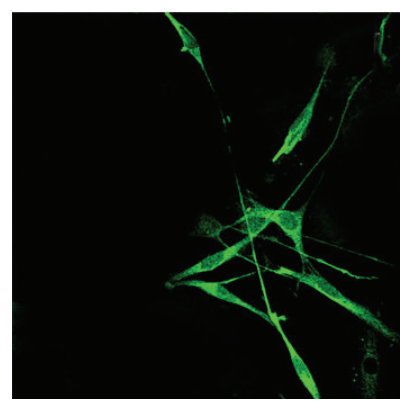

(n)

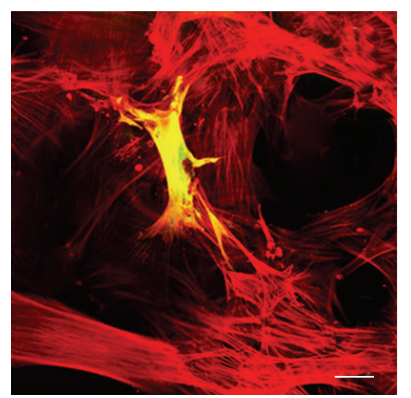

(r)

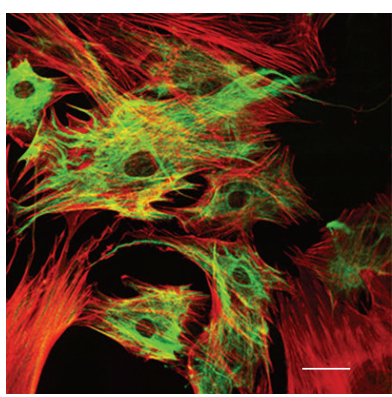

(c)

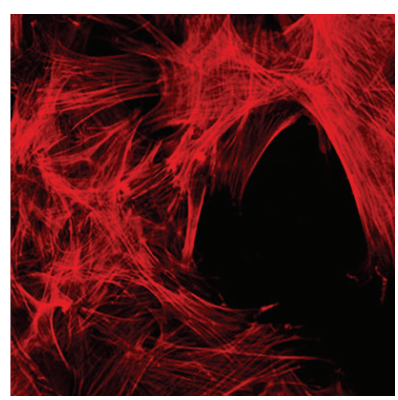

(g)

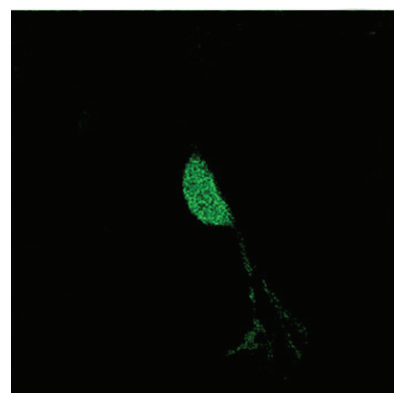

(k)

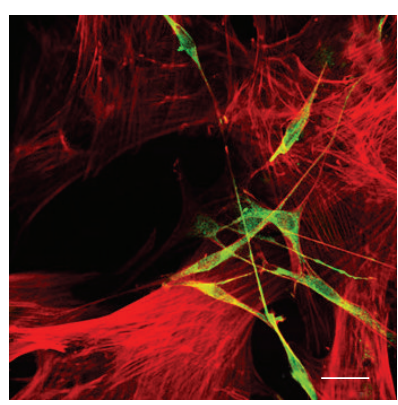

(o)

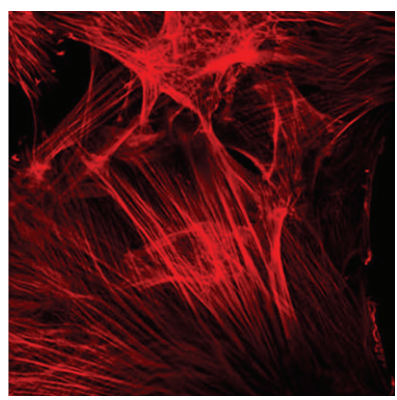

(s)

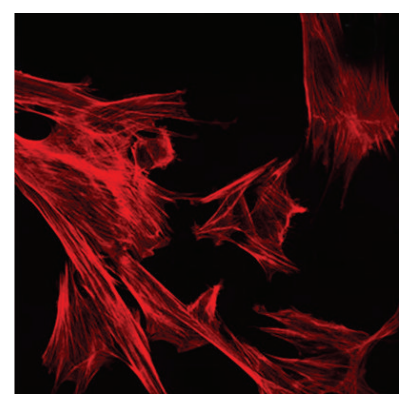

(d)

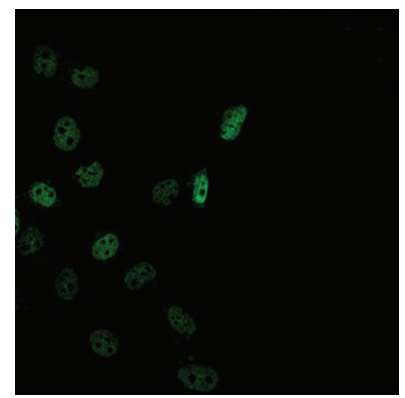

(h)

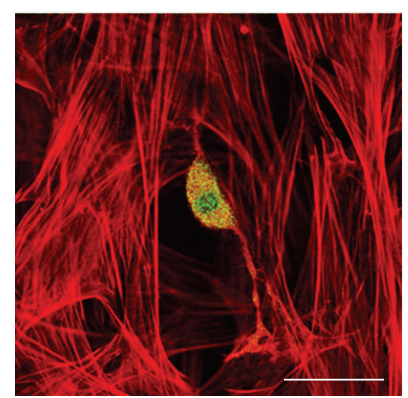

(1)

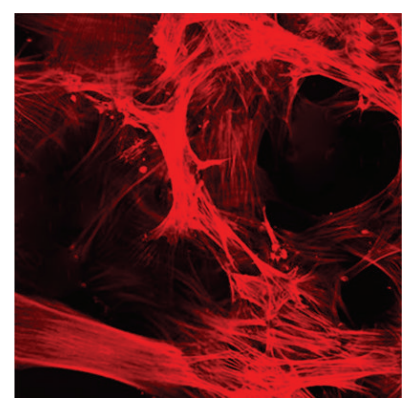

(p)

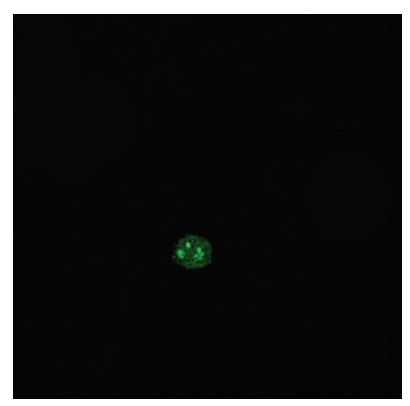

(t)

FIgURE 5: Continued. 


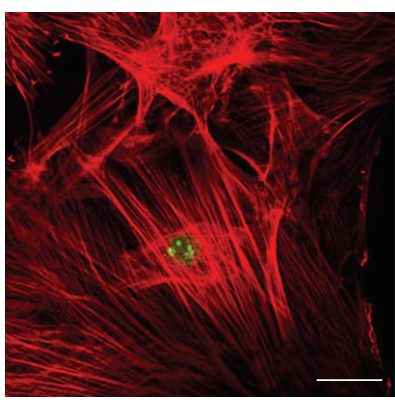

(u)

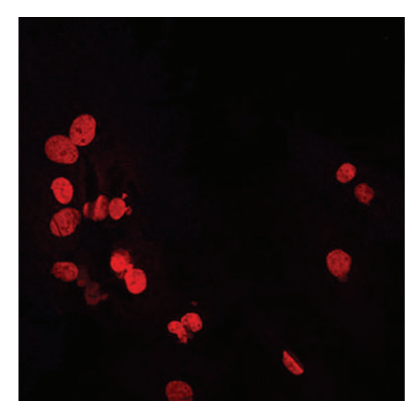

(v)

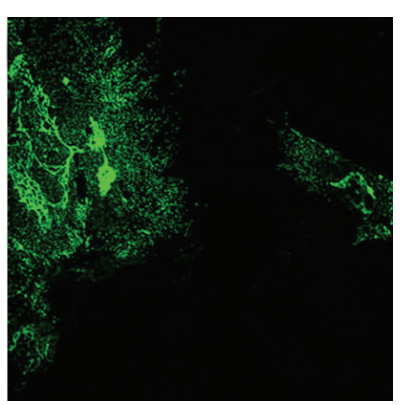

(w)

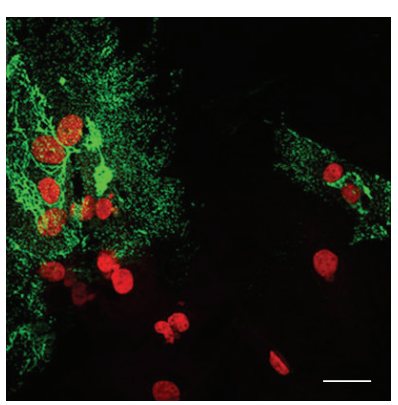

(x)

FIGURE 5: Spontaneous expression of neural and mesengenic markers by undifferentiated rMSCs (P4) after 14 days of culture. Phalloidin staining labeled actin filaments in red ( $a, d, g, j, m, p$, and s), and neural and mesengenic markers were labelled in green (b, e, h, k, n, $q$, t, and $\mathrm{w}$ ). Propidium iodide labeled nuclei in red (v), used instead of phalloidin for a better labelling visualization after superimposition, being OPN expressed as dots. Most of cells were, respectively, positive for nestin (b) and $\beta$ III tubulin (e). Numerous cells were NeuN positive with nuclear localization (h). Few cells were, respectively, positive for GFAP (n) and OPN (w). Very few cells were, respectively, positive for NF (k), S100 (q), and PPAR $\gamma(\mathrm{t})$. Merges (c, f, i, l, o, r, u, and x). Bars: $50 \mu \mathrm{m}$.

50-70\% of rMSCs were positive for the marker (Figure 5(e)). At P8, P16, and P24 rMSCs expressing $\beta$ III tubulin were reduced, and they were about $10-30 \%$. At P40, $\beta$ III-tubulin expression decreased drastically $(<1 \%)$ becoming negative at P80 (Table 1).

The late neuronal marker NeuN (Figure 5(h)) was expressed by rMSCs mostly at P0, P2, and P4 (about 30$50 \%$ of cells), and its expression decreased progressively at the subsequent passages. At P40 and P80 no cells were NeuN positive (Table 1).

The late neuronal marker neurofilament (NF) was not expressed by rMSCs at any culture time examined at P0 and P1. At P2, P4, P8, P16, and P24, we observed no more than $1 \%$ of cells that were NF positive (Figure $5(\mathrm{k})$ ). At P40 and P80 this neuronal marker was not expressed by any cell (Table 1).

The glial marker GFAP was mainly expressed by rMSCs at P0, where about $50 \%$ of cells were GFAP positive. From $\mathrm{P} 1$ to the subsequent passages GFAP expression decreased progressively, and at P40 and P80 no rMSCs expressed this marker (Table 1). In rMSC cultures, GFAP-positive cells were characterized by both a large flattened morphology (Figure 6(e)) or a more elongated one (Figure $5(\mathrm{n})$ ) in almost the same number.

The expression of the glial marker S100 was evident at P0 and $\mathrm{P} 1$ and was extended, respectively, to about $10 \%$ and less than $5 \%$ of cells. At P2, P4, P8, P16, and P24 the percentage of positive rMSCs decreased to less than 1\% (Figure 5(q)). At P40 and P80 no S100-positive cells were observed in our rMSC cultures (Table 1).

From the earliest passages ( $\mathrm{P} 0, \mathrm{P} 1$, and $\mathrm{P} 2)$, the adipogenic marker PPAR $\gamma 2$ was expressed by only a few cells $(<5 \%)$. The percentage of cells that expressed PPAR $\gamma 2$ was further reduced from $\mathrm{P} 4$ to subsequent passages where the expression of this protein was observed in less than $1 \%$ of cells (Figure 5(t)). At p40 some rare cells were PPAR $\gamma 2$ positive, and at P80 no cells expressing this marker were observed (Table 1). PPAR $\gamma 2$-positive rMSCs were often characterized by actin filaments that were not well-organized, unlike the majority of cells that presented a typical fibroblastic-like morphology.

At P0 and P1 the osteogenic marker osteopontin (OPN) was expressed by about 10-15\% of cells that were frequently clustered. At P2 we observed more clustered OPN-positive cells. From P4 to subsequent passages (Figure 5(w)), the number of cells expressing OPN decreased progressively (about $10 \%$ at $\mathrm{P} 4$ and $\mathrm{P} 8$, and about $5 \%$ at P16), and at P24 and $\mathrm{P} 40$ a very small number of cells positive for this protein were observed. At P80 no OPN-positive cells were present in our rMSC cultures (Table 1). The osteogenic marker osteocalcin (OC) was negative at each passage and time examined (Table 1).

\subsection{Coexpression of Neural Markers by Undifferentiated} rMSCs. At different passages and at different times (see Section 2), in undifferentiated rMSCs, we examined the coexpression of nestin with neural and mesengenic markers by means of double-labeling immunofluorescence experiments. In all culture passages examined, the coexpression of nestin with the mesengenic markers PPAR $\gamma 2$ and OPN was not observed except for P1, but in a very low percentage of cells $(<1 \%)$. Nestin coexpression with the early neuronal marker $\beta$ III tubulin (Figures 6(a)-6(c)) and with the glial marker GFAP (Figures 6(d)-6(f)) was observed at each passage and culture time examined except for P40 and P80. In contrast, nestin coexpression with the late neuronal marker NeuN was observed at the earliest passages ( $\mathrm{P} 0, \mathrm{P} 1$, and $\mathrm{P} 2$ ) and, from $\mathrm{P} 4$ to subsequent passages, we did not observe any nestin-positive/NeuN-positive rMSCs (Figures 6(g)-6(i)). In all the coexpression experiments performed, rMSCs that were positive only for one of the examined markers were also evident.

\subsection{Evaluation of Neural Marker Expression by Immunoblot-} ting. Nestin, $\beta$ III-tubulin, NeuN, and GFAP expression in undifferentiated rMSCs was evaluated through immunoblotting analysis. Concerning the neuroprogenitor marker 


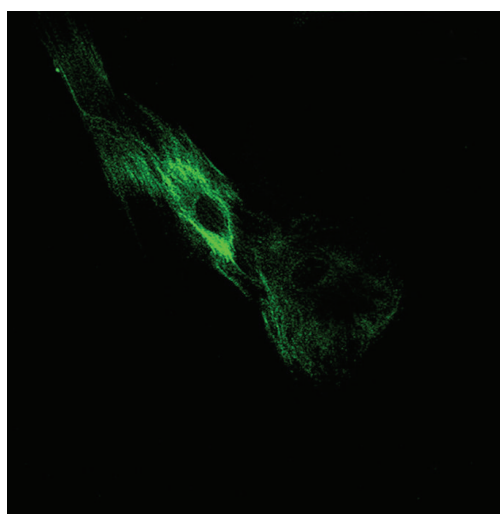

(a)

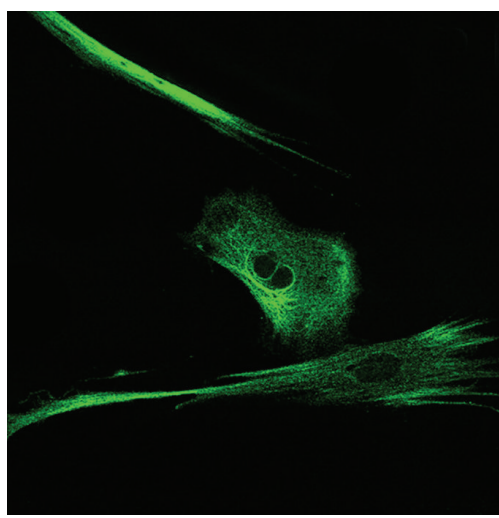

(d)

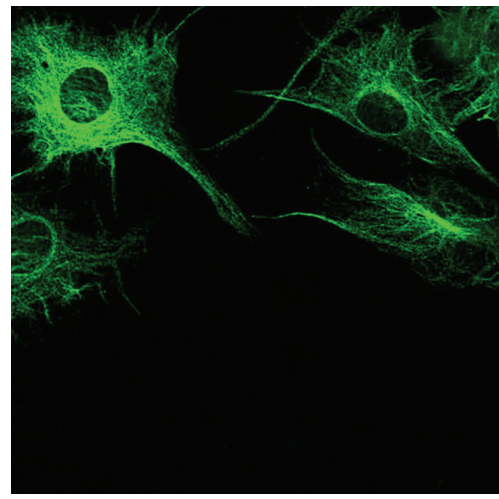

(g)

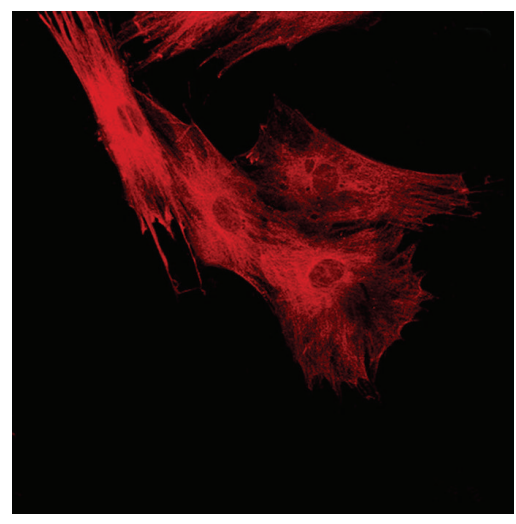

(b)

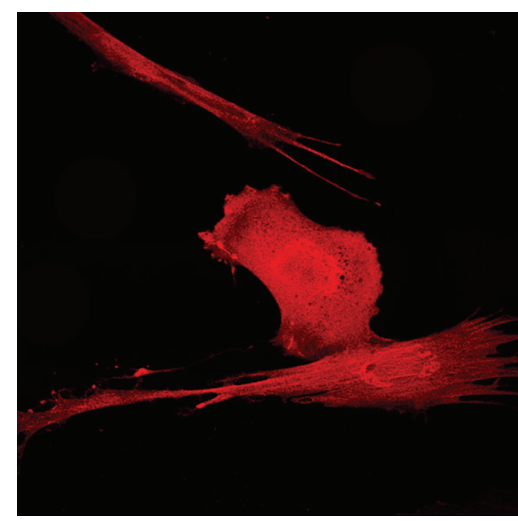

(e)

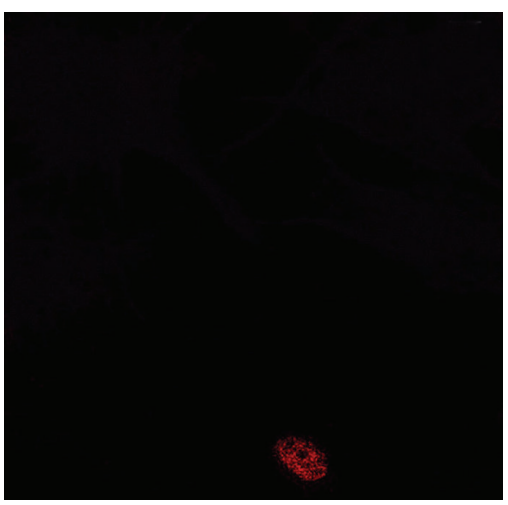

(h)

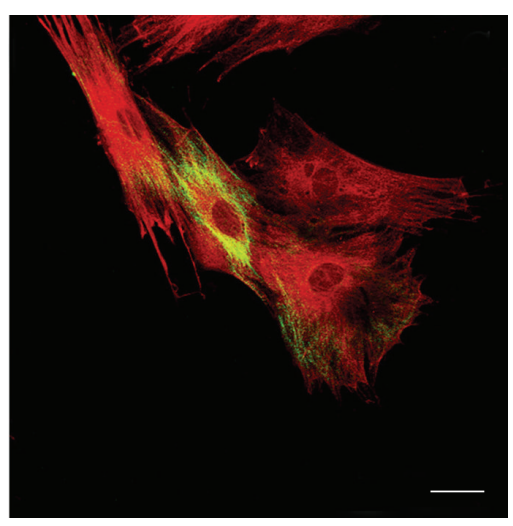

(c)

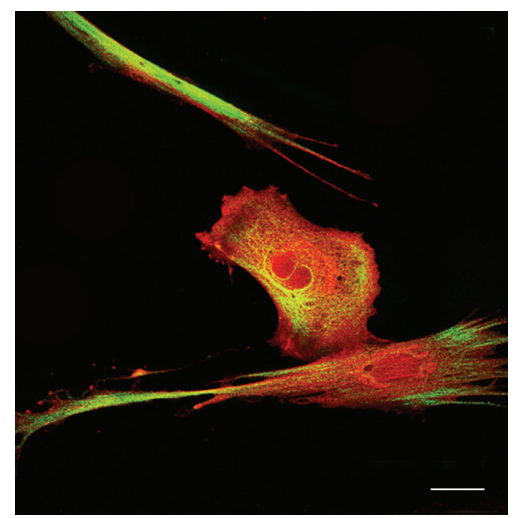

(f)

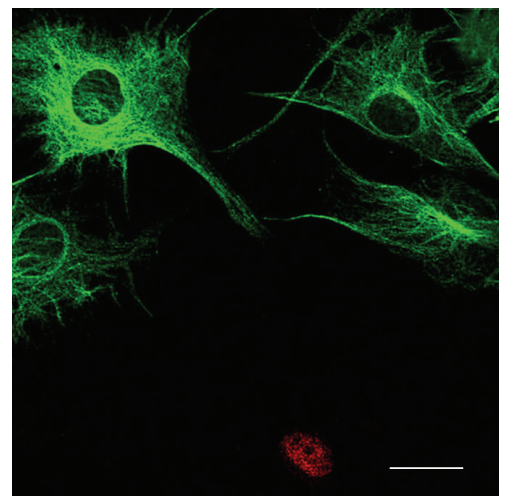

(i)

FIGURE 6: Spontaneous coexpression of neural markers by undifferentiated rMSCs (P4) after 14 days of culture. Nestin was labelled in green (a, d, and g), and the other neural markers in red: $\beta$ III-tubulin (b); GFAP (e); NeuN (h). Merges (c, f, and i). Bars: $50 \mu \mathrm{m}$.

nestin, two bands corresponding to 200 and $220 \mathrm{kDa}$ molecular weight were evident (Figure 7(a)). A band corresponding to a predicted $50 \mathrm{kDa}$ molecular weight was evident, respectively for $\beta$ III-tubulin and GFAP immunoblotting (Figure $7(\mathrm{~b})$ ). Concerning NeuN (nuclear extracts), the immunoblot showed two major NeuN species at $45-50 \mathrm{kDa}$ and additional reactive bands at $\sim 66 \mathrm{kDa}$ and between 70 and $90 \mathrm{kDa}$ in accordance with the literature data [27] (Figure 7(b)). Actin was used as loading control (Figures 7(a) and $7(\mathrm{~b}))$.

\section{Discussion}

In this study we have demonstrated that rMSCs, in the absence of any differentiative agent, are able to spontaneously express neural markers. This finding is not new, but, compared to the literature data, our study is more extensive and thorough in terms of the panel of markers and number of passages. Our results show that the percentage of rMSCs expressing neural markers depends on the culture passage. In fact, at late passages, in which cells are not 


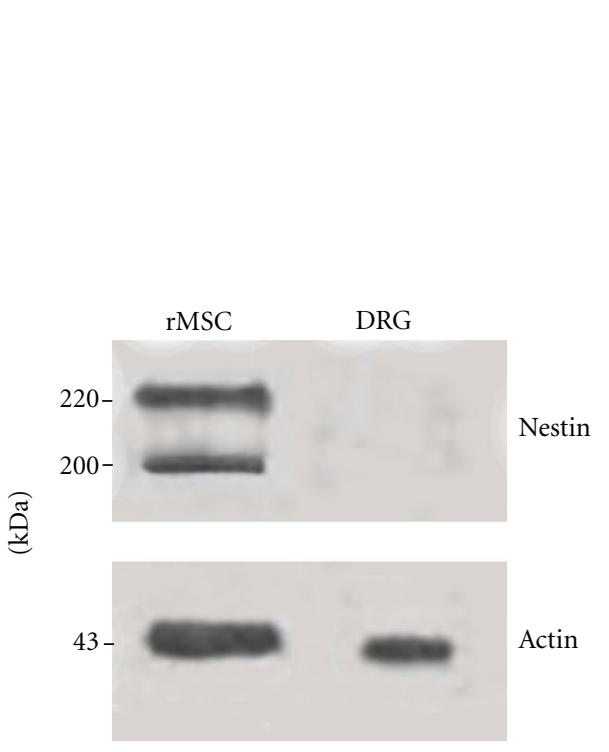

(a)

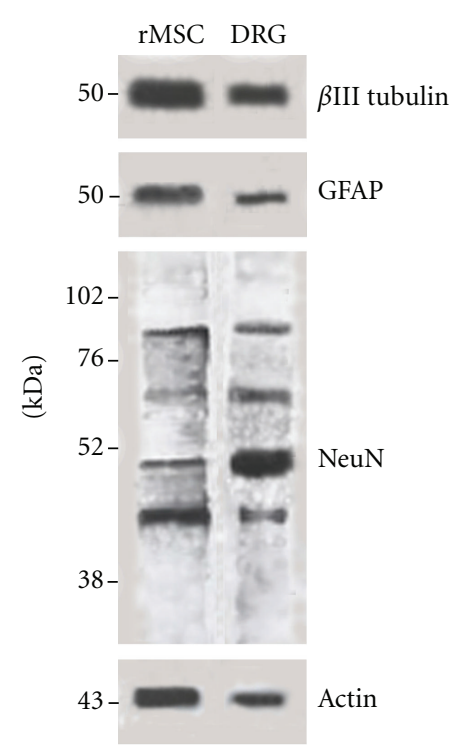

(b)

FIGURE 7: rMSC expression of neural markers by immunoblotting. Protein extracts $(15 \mu \mathrm{g})$, from rMSC or DRG cultures, were separated by $7.5 \%$ SDS-PAGE (a) or 13\% SDS-PAGE (b) and transferred to a nitrocellulose membrane. Membranes were, respectively, blotted with anti-nestin, anti- $\beta$ III-tubulin, anti-GFAP, and anti-NeuN antibodies. Two bands corresponding to 200 and $220 \mathrm{kDa}$ molecular weight were evident for nestin (a), a $50 \mathrm{kDa}$ band for $\beta \mathrm{III}$ tubulin and GFAP (b) immunoblottings. The NeuN blot shows two major bands at $45-50 \mathrm{kDa}$ and additional reactive bands at $\sim 66 \mathrm{kDa}$ and between 70 and $90 \mathrm{kDa}(\mathrm{b})$. Actin was used as loading control ( $\mathrm{a}$ and b).

senescent and maintained the MSC immunophenotype, the expression of neural markers decreases or is absent in comparison with early passages. On the other hand, the spontaneous expression of mesengenic differentiation markers, in rMSCs, is very low or absent at all passages examined.

The ability of undifferentiated rMSCs to express neural proteins confirms that MSCs are multidifferentiated cells [28] and suggests that rMSCs have a wide differentiation potential due to an intrinsic plasticity [29]. rMSCs that spontaneously express neural markers retain a mesenchymal morphology, and no neural phenotypes are observed. This finding excludes the possibility that the expression of neural markers by rMSCs may be a consequence of spontaneous neural differentiation.

The biological origin of MSCs may account for the expression of neural markers by undifferentiated rMSCs. It has been demonstrated that, during embryonic development, MSCs are generated from the neural crest and that neural crest-derived MSCs may persist in adult bone marrow [30, 31]. If the neural crest origin can explain the spontaneous expression of neural markers, this expression should be limited to an MSC subset, which was not the case in our rMSC cultures. An alternative explanation has been proposed [9]. It has been hypothesized that neuronal gene expression in MSCs could be due to the release of neuronrestrictive silencer factor (NRSF) involved in the repression of the neuron-specific gene in nonneuronal cells [32]. This hypothesis could account for the rMSCs' spontaneous expression of neuronal markers, but not for the expression of nestin and glial markers.
The expression of nestin is considered to be one of the initial steps in the MSC progression to neural lineage. We have found that, in the presence of serum, nestin is expressed by a high percentage of undifferentiated rMSCs even at very early culture passages. In contrast, previous publications have reported that the presence of serum inhibited nestin expression and that, only after serum starvation, was an enrichment in nestin-expressing cells observed $[11,13]$. This discrepancy could be explained in terms of differences in the rat strains used to isolate MSCs and different methods of cell isolation and culture conditions.

In the literature the existence of MSC subpopulations has been proposed $[8,33,34]$. In agreement with this, we have found that the overall population of rMSCs is not homogeneous but is composed of distinct subpopulations that, in our study, are characterized by the expression of different neural markers. Neuronal and glial markers are coexpressed or not with the neural progenitor marker nestin suggesting the existence, respectively, of immature and mature neurally fated rMSC subpopulations.

In conclusion, we have demonstrated the spontaneous expression of neural markers by undifferentiated rMSCs, thereby supporting the concept of MSCs as multidifferentiated cells. Moreover, the presence of distinct rMSC subpopulations suggests that the controversial results regarding MSC neuronal differentiation could derive from the use of the whole population of MSCs, whereas the use of a neutrally fated subpopulation of MSCs could optimize their differentiation. The possibility of linking a specific MSC subpopulation with a specific neural lineage provides 
a framework for optimizing future transplantation studies aimed at treating neurodegenerative diseases.

\section{Acknowledgment}

The authors are grateful to Dr. E. Genton for language assistance.

\section{References}

[1] G. Brooke, M. Cook, C. Blair et al., "Therapeutic applications of mesenchymal stromal cells," Seminars in Cell and Developmental Biology, vol. 18, no. 6, pp. 846-858, 2007.

[2] Y. L. Si, Y. L. Zhao, H. J. Hao, X. B. Fu, and W. D. Han, "MSCs: biological characteristics, clinical applications and their outstanding concerns," Ageing Research Reviews, vol. 10, no. 1, pp. 93-103, 2011.

[3] O. Bugos, M. Bhide, and N. Zilka, "Beyond the rat models of human neurodegenerative disorders," Cellular and Molecular Neurobiology, vol. 29, no. 6-7, pp. 859-869, 2009.

[4] B. Zavan, C. Giorgi, G. P. Bagnara, V. Vindigni, G. Abatangelo, and R. Cortivo, "Osteogenic and chondrogenic differentiation: comparison of human and rat bone marrow mesenchymal stem cells cultured into polymeric scaffolds," European Journal of Histochemistry, vol. 51, no. 1, pp. 1-8, 2007.

[5] D. Foudah, S. Redaelli, E. Donzelli et al., "Monitoring the genomic stability of in vitro cultured rat bone-marrowderived mesenchymal stem cells," Chromosome Research, vol. 17, no. 8, pp. 1025-1039, 2009.

[6] E. Karaoz, A. Aksoy, S. Ayhan, A. E. SarIboyacI, F. Kaymaz, and M. Kasap, "Characterization of mesenchymal stem cells from rat bone marrow: ultrastructural properties, differentiation potential and immunophenotypic markers," Histochemistry and Cell Biology, vol. 132, no. 5, pp. 533-546, 2009.

[7] B. Neuhuber, G. Gallo, L. Howard, L. Kostura, A. Mackay, and I. Fischer, "Reevaluation of in vitro differentiation protocols for bone marrow stromal cells: disruption of actin cytoskeleton induces rapid morphological changes and mimics neuronal phenotype," Journal of Neuroscience Research, vol. 77, no. 2, pp. 192-204, 2004.

[8] Z. Lei, L. Yongda, M. Jun et al., "Culture and neural differentiation of rat bone marrow mesenchymal stem cells in vitro," Cell Biology International, vol. 31, no. 9, pp. 916-923, 2007.

[9] G. F. Barnabé, T. T. Schwindt, M. E. Calcagnotto et al., "Chemically-induced RAT mesenchymal stem cells adopt molecular properties of neuronal-like cells but do not have basic neuronal functional properties," PLOS ONE, vol. 4, no. 4, Article ID e5222, 2009.

[10] M. G. Thomas, L. Stone, L. Evill, S. Ong, M. Ziman, and L. Hool, "Bone marrow stromal cells as replacement cells for Parkinson's disease: generation of an anatomical but not functional neuronal phenotype," Translational Research, vol. 157, no. 2, pp. 56-63, 2011.

[11] S. Wislet-Gendebien, P. Leprince, G. Moonen, and B. Rogister, "Regulation of neural markers nestin and GFAP expression by cultivated bone marrow stromal cells," Journal of Cell Science, vol. 116, no. 16, pp. 3295-3302, 2003.

[12] P. Lu, A. Blesch, and M. H. Tuszynski, "Induction of bone marrow stromal cells to neurons: differentiation, transdifferentiation, or artifact?" Journal of Neuroscience Research, vol. 77, no. 2, pp. 174-191, 2004.

[13] A. P. Croft and S. A. Przyborski, "Formation of neurons by non-neural adult stem cells: potential mechanism implicates an artifact of growth in culture," Stem Cells, vol. 24, no. 8, pp. 1841-1851, 2006.

[14] G. E. Rooney, L. Howard, T. O’Brien, A. J. Windebank, and F. P. Barry, "Elevation of cAMP in mesenchymal stem cells transiently upregulates neural markers rather than inducing neural differentiation," Stem Cells and Development, vol. 18, no. 3, pp. 387-398, 2009.

[15] G. Nicolini, R. Rigolio, A. Scuteri et al., "Effect of transresveratrol on signal transduction pathways involved in paclitaxel-induced apoptosis in human neuroblastoma $\mathrm{SH}$ SY5Y cells," Neurochemistry International, vol. 42, no. 5, pp. 419-429, 2003.

[16] F. Ronca, S. L. Chan, and V. C. Yu, "1-(5-Isoquinolinesulfonyl)-2-methylpiperazine induces apoptosis in human neuroblastoma cells, SH-SY5Y, through a p53-dependent pathway," Journal of Biological Chemistry, vol. 272, no. 7, pp. 4252-4260, 1997.

[17] M. Dominici, K. Le Blanc, I. Mueller et al., "Minimal criteria for defining multipotent mesenchymal stromal cells. The International Society for Cellular Therapy position statement," Cytotherapy, vol. 8, no. 4, pp. 315-317, 2006.

[18] C. D. Katsetos, A. Legido, E. Perentes, and S. J. Mörk, "Class III $\beta$-tubulin isotype: a key cytoskeletal protein at the crossroads of developmental neurobiology and tumor neuropathology," Journal of Child Neurology, vol. 18, no. 12, pp. 851-866, 2003.

[19] R. J. Mullen, C. R. Buck, and A. M. Smith, "NeuN, a neuronal specific nuclear protein in vertebrates," Development, vol. 116, no. 1, pp. 201-211, 1992.

[20] M. K. Lee and D. W. Cleveland, "Neuronal intermediate filaments," Annual Review of Neuroscience, vol. 19, pp. 187217, 1996.

[21] L. F. Eng, R. S. Ghirnikar, and Y. L. Lee, "Glial fibrillary acidic protein: GFAP-thirty-one years (1969-2000)," Neurochemical Research, vol. 25, no. 9-10, pp. 1439-1451, 2000.

[22] R. Donato, "S100: a multigenic family of calcium-modulated proteins of the EF-hand type with intracellular and extracellular functional roles," International Journal of Biochemistry and Cell Biology, vol. 33, no. 7, pp. 637-668, 2001.

[23] J. Sodek, B. Ganss, and M. D. McKee, “Osteopontin," Critical Reviews in Oral Biology and Medicine, vol. 11, no. 3, pp. 279303, 2000.

[24] P. V. Hauschka, "Osteocalcin: the vitamin K-dependent Ca2+binding protein of bone matrix," Haemostasis, vol. 16, no. 3-4, pp. 258-272, 1986.

[25] E. D. Rosen and B. M. Spiegelman, "PPAR $\gamma$ : a nuclear regulator of metabolism, differentiation, and cell growth," Journal of Biological Chemistry, vol. 276, no. 41, pp. 3773137734, 2001.

[26] C. Wiese, A. Rolletschek, G. Kania et al., "Nestin expressiona property of multi-lineage progenitor cells?" Cellular and Molecular Life Sciences, vol. 61, no. 19-20, pp. 2510-2522, 2004.

[27] B. K. Dredge and K. B. Jensen, "NeuN/Rbfox3 Nuclear and Cytoplasmic isoforms differentially regulate alternative splicing and nonsense-mediated decay of Rbfox2," PLoS ONE, vol. 6, no. 6, Article ID e21585, 2011.

[28] N. R. Blondheim, Y. S. Levy, T. Ben-Zur et al., "Human mesenchymal stem cells express neural genes, suggesting a neural predisposition," Stem Cells and Development, vol. 15, no. 2, pp. 141-164, 2006.

[29] T. Tondreau, L. Lagneaux, M. Dejenefle et al., "Bone marrowderived mesenchymal stem cells already express specific neural proteins before any differentiation," Differentiation, vol. 72, no. 7, pp. 319-326, 2004. 
[30] Y. Takashima, T. Era, K. Nakao et al., "Neuroepithelial cells supply an initial transient wave of MSC differentiation," Cell, vol. 129, no. 7, pp. 1377-1388, 2007.

[31] S. Morikawa, Y. Mabuchi, K. Niibe et al., "Development of mesenchymal stem cells partially originate from the neural crest," Biochemical and Biophysical Research Communications, vol. 379, no. 4, pp. 1114-1119, 2009.

[32] C. J. Schoenherr and D. J. Anderson, "The neuron-restrictive silencer factor (NRSF): a coordinate repressor of multiple neuron-specific genes," Science, vol. 267, no. 5202, pp. 1360$1363,1995$.

[33] F. M. J. Lamoury, J. Croitoru-Lamoury, and B. J. Brew, "Undifferentiated mouse mesenchymal stem cells spontaneously express neural and stem cell markers Oct-4 and Rex1," Cytotherapy, vol. 8, no. 3, pp. 228-242, 2006.

[34] L. Fu, L. Zhu, Y. Huang, T. D. Lee, S. J. Forman, and C. C. Shih, "Derivation of neural stem cells from mesenchymal stem cells: evidence for a bipotential stem cell population," Stem Cells and Development, vol. 17, no. 6, pp. 1109-1121, 2008. 

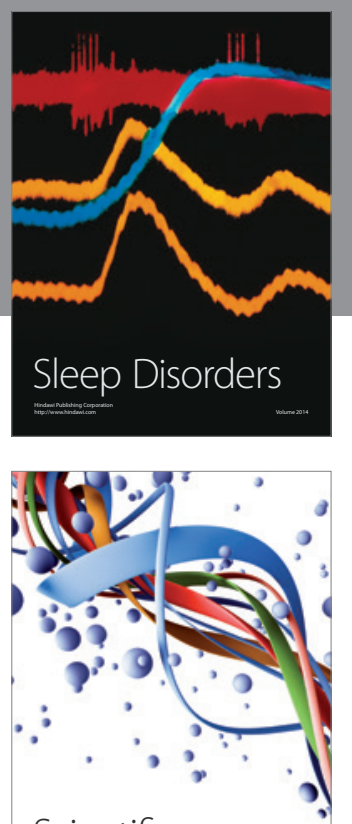

Scientifica
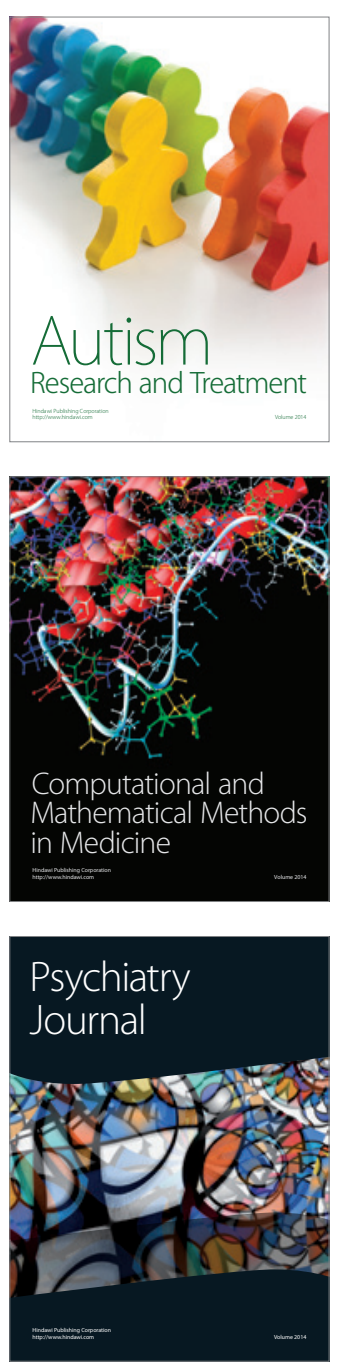
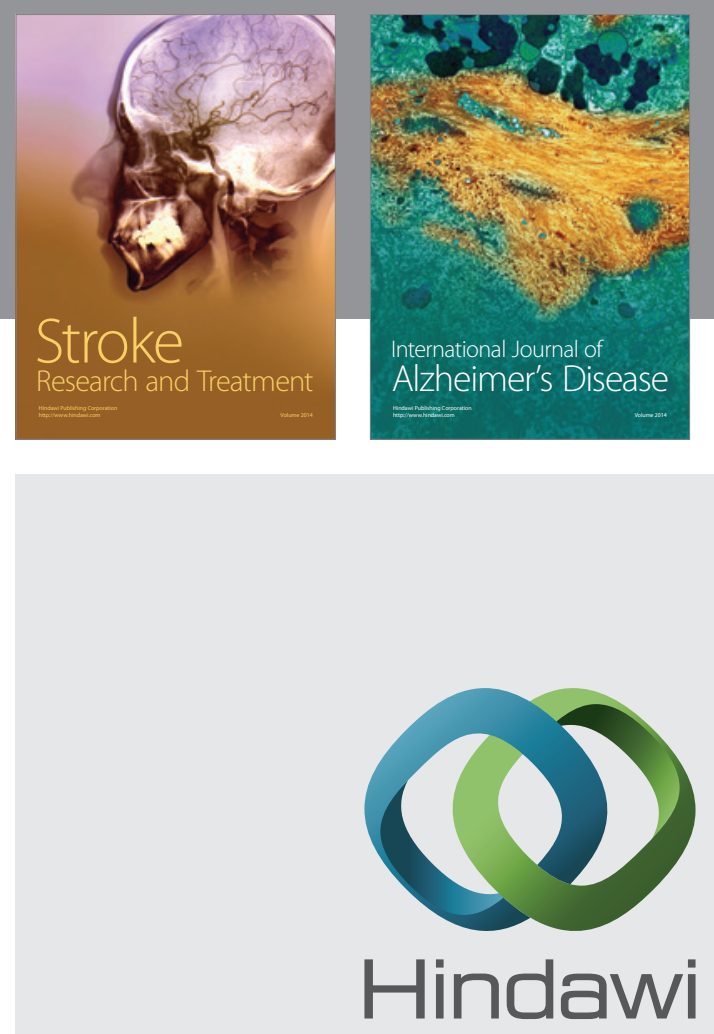

Submit your manuscripts at

http://www.hindawi.com
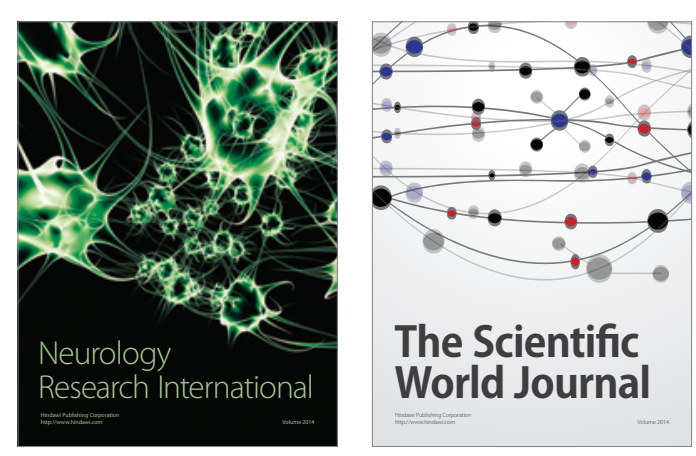

The Scientific World Journal

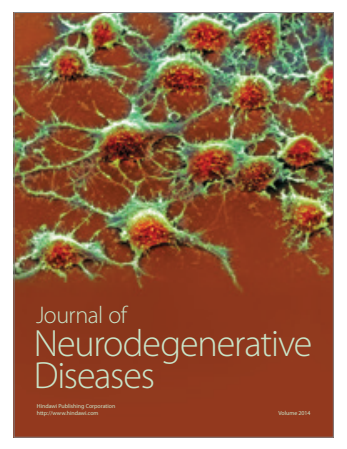

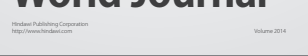

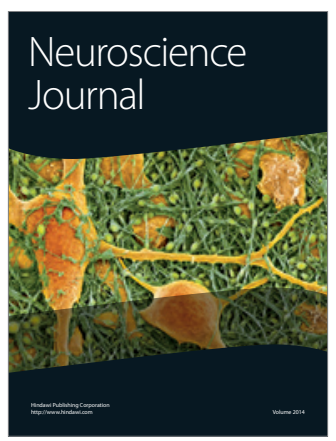

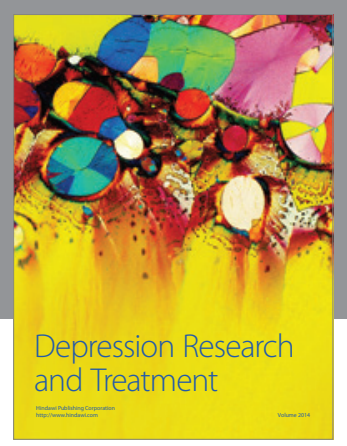
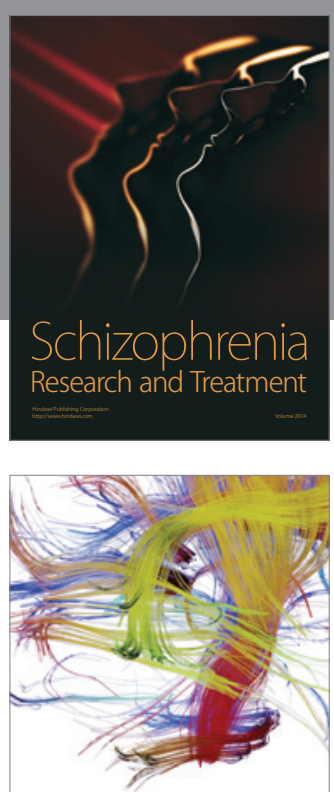

Brain Science

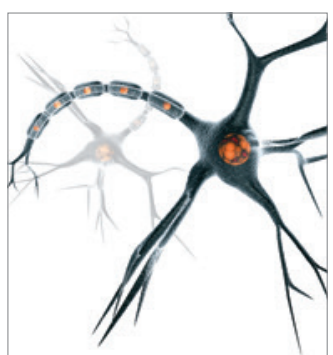

Neural Plasticity
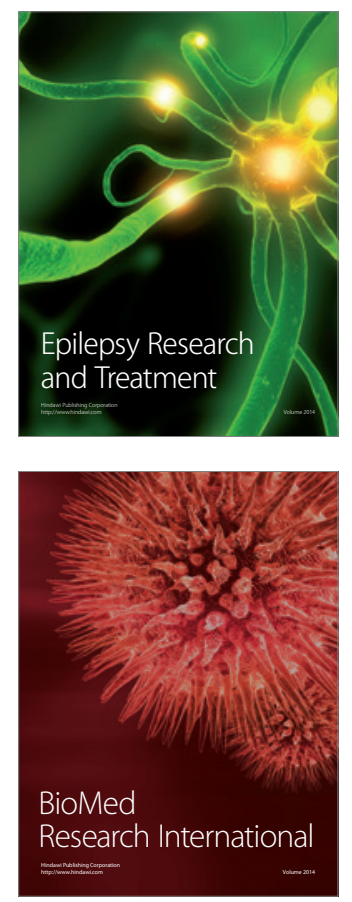

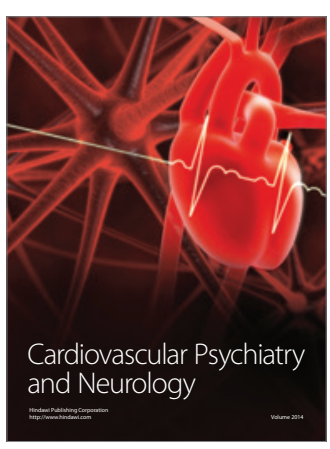

Parkinson's

Disease
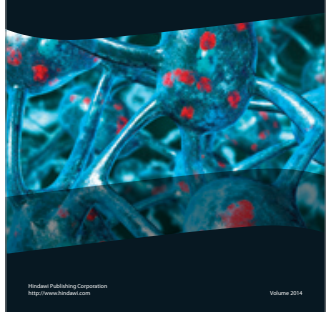